\title{
Precision medicine and traditional chinese medicine of dialogue
}

\author{
Xin Lou ${ }^{1}$, Ming Bai ${ }^{1}$ and Mingsan Miao ${ }^{1, a}$ \\ ${ }^{1}$ University of Traditional Chinese Medicine, yaolixueke, 450046 zhengzhou, China
}

\begin{abstract}
The precision medicine is more precise individualized medicine, based on the patient's genes or physiological to formulate the specific treatment plan, for the realization of individualized treatment of various diseases to provide valuable information. But with the progress of modern science and technology, modern medicine dependence on medical instruments are too serious, traditional ways are gradually forgotten.If the machine depends on the instrument test results too serious which don't combined with the actual diagnosis, the cause of misdiagnosis, so we should pay attention to the overall analysis of diseases and systematic diagnosis and examination, use of the overall treatment concept traced back to find the cause of Traditional Chinese Medicine, finally decide to select a best treatment plan. We should use the dialectical attitude to look at the precise medical.Not blindly requirements according to the road of precision medicine of Traditional Chinese Medicine to go, to shine in himself field, form of self characteristic of Traditional Chinese Medicine.Can learn some of the advantages of accurate concept, the good and rejecting the bad, hope the Traditional Chinese Medicine in the modern environment more walk more far.
\end{abstract}

The concept of precision medical is drugs in the future will be customized for each individual or a small group of people, At present using mass production of a class of drugs, and to all the people of the same kind of disease take the same medicine situation will be gradually eliminated.It is more precise individualized medicine, based on a genetic or biological treatment to customize.As follow is the Specific steps:First, in patients with a large number of individuals and genome sequencing to build a huge medical data information database, and then through the analys is of the genetic information of the comparis on of different individuals, to further understand the common cause and individual cause of various diseases, in the last developed for particular patients with specific diseases pathogenic gene targeted drugs and therapies, of course, also for personalized preventive care for healthy people.Each patient has a "card", Doctor "swipe" identification information, sympto matic to people; To see a doctor and treating according to each person's genome, take medication as the main body of the existing medical reform, thus will influence and change the future of medical care, drug development and use.Will also find more and more treatments for cancer and for the realization of individualized treatment of various diseas es to provide valuable information.

With the concept of precision medical widely promoted and in clinical application,the effect of the play is also more and more obvious, especially in the cancer therapy has made big contribution, but has some concrete problems to be solved on the clinical application, precision medical has some farfetched in the face of the whole concept and theory of Traditional Chinese Medicinal and the theory of property of chinese herbs, medicinal herbs of warm, hot, cold, cool, cannot be explained by the

${ }^{\mathrm{a}}$ Corresponding author: miaomingsan@163.com. 
concept of precision medicine, in the face of these problems we need to correct view precision med icine, reasonable applied to clinical therapy.

\section{The application of precision medical}

Precision medical plays a significant role in the treatment of diseases, such as heart cerebrovascular disease, cancer, neurodegenerative diseases and so on.

\subsection{Precise application in cardiovascular medicine}

In recent years, on the basis of the gene sequencing of prec ision medical get rapid development, heart vascular disease, clinical go up to with hypertrophic cardio myopathy, sudden cardiac death, such as genetic hypertension monogenic disease genetic cardiovascular dis ease has through genetic testing for early diagnosis, early warning and targeted therapy, pathogenic gene mutation carriers and their families for genetic screening, genetic block, predict curative effect, individualized treatment, cardiovascular field will beco me the new direction of prec ision medical.

\subsection{Precision medical applications in cancer}

Presently, precision medical in the treatment of cancer get good progress and results.For example, there are more and more patients with breast cancer, lung cancer, colorectal cancer, and me lano ma are applied precise treatment to cure the disease and tested for genome in treatment, doctors can according to each person's genome differences make the best treatment plan.Breast cancer after decades of research, for example, has been divided into 10 categories, according to a new system of classification and diagnosis, clinicians can according to different category, take accurate treatment plan to make possible a variety of breast cancer to cure.

\subsection{Precision medical applications in neurodegenerative diseases}

Neurodegenerative disease is a kind of complex diseases, including Alzheimer's disease, Parkinson's disease, Amyotrophic Amyotrophic lateral sclerosis, such as its pathogenesis, early diagnosis difficult, complex causes the lack of treatment, the serious influence the patient's quality of life and work.Currently, neurodegenerative diseases "precision medical" just getting started, there are a lot of work to be carried out.For example, the use of genomics, proteomics, metabolo mics technology such as finding more disease related gene, molecular Marker, and by adopting the technology of pharmacogenomics, the development of individualized targeted drugs, "tailored" to use drugs, to achieve the ultimate neurodegenerative diseases, to promote the "precision medical treatment", better service for patients with medical treatment.In early diagnosis, need to be patient of genomics, proteomics, metabolomics etc information analysis, to fully as sess risk of patients; In the treatment of disease, patients with combined drug genome information needed, personalized treatment options.

\subsection{Precision medical problems in the treatment of cancer}

In tumor treatment, for example, has now been confirmed in cancerous cells exist in the process of a large number of genetic mutations, suggesting that most of the mutations, there is no significant effect on tumor occurrence and evolution, but how to determine and accurately interpret these key mutations will not be easy;And the tumor cells in the process of treatment is not completely passive, tumor cells can evolve through natural selection, or evolution, single drug treatment all cannot kill tumor cells is also one of the reasons. This is because the tumor is the result of the interaction between genes and environment, environmental factors caused by tumor cell gene structure and function of change, and ultimately make tumor cells to better adapt to the environment. Only fro $m$ the tu mor on the one hand, 
accurate treatment unsolved problem existed, is not to remove a lesion can be cured.Therefore, the precise treatment in clinical medical treatment still has a long way to go.

\section{5 for the treatment of using precise idea of thinking}

With the implementation of the concept of "precision medicine", it should be optimistic about the concept, but also should be questioned.Questioning the idea comes from the understanding of the disease itself, That is, whether the problem can be accurate diagnosis and treatment.Until today in development of medicine, It can cure diseases are numbered, Most chronic diseases are usually not just one of the body's problems, but one or more of the system's problems, and may be the whole body of one or more of the system is out of the question. What is more important to human health are the result of complex multi-factor comprehensive effect, at the same time, because each person's physical quality is different, each kind of disease according to the specific circumstances of individual patients have different manifestations. So you should see, most of the complexity of the disease is caused by a single factor. Therefore, because the comple xity of the disease itself, which makes the precise concept more stay at the ideal level.

\section{Precision medicine in the application of traditional chinese medicine}

Figures and tables, as originals of good quality and well contrasted, are to be in their final form, ready for reproduction, pasted in the appropriate place in the text. Try to ensure that the size of the text in your figures is approximately the same size as the main text (10 point). Try to ensure that lines are no thinner than 0.25 point.

\subsection{Characteristics of traditional chinese medicine thinking - the whole}

The whole concept has a pivotal position in the Traditional Chinese Medicine, to observe and explore the relationship between the human body and the human body and the external environment and the clinical diagnosis and treatment of diseases has an important guiding significance, believes that the human body is an organic whole, constitute the various parts of the human body in the physiological, pathological are inseparable, mutual influence; At the same time we also recognizes the unity of body and the environment. The unity of internal and external environment, the body's own integrity of the idea, referred to as the overall concept. In some respects, Human beings live in nature, there exist necessary conditions for the survival of human beings. At the same time, the changes of nature will directly or indirectly affect the physiological or pathological state of the human body, and the body will react accordingly. Under the influence of the overall concept, Traditional Chinese Medicine only consider the overall accuracy is the true precision!

\subsection{Characteristics of traditional chinese medicine treatment-syndrome differentiation}

Analysis, identification of disease syndrome is a syndrome differentiation. Identify the cause of the disease, the nature of the site, and then determine where to judge; The disease syndromes performance varied, pathological changes is very complex, lesions with different time, space and different individuals have different influence on the changes of disease. By syndrome differentiation, distinguish between the phenomenon and the essence of the disease, you can seek the treatment. According to the different time of onset, different individual characteristics, location and time to be patient, because the treatment of the person. According to different causes, illness, disease, pathogenesis to determine the specific treatment methods, and then choose an effective drug, which can also be said to be a precise expression. 


\subsection{Characteristics of Traditional Chinese Medicine - compatibility}

Etiology is not unique, the only precise is the narrow sense of precision, For the cause of the compound is accurate. Zhongjing formula, such as less of its ingredients, but fujin made excellent compatibility, if we change the amount of a medicine in the formula, it is possible to result in great changes of prescription effect.

In the case of Guizhi decoction, the party by $90 \mathrm{~g}$ cassia twig, peony, licorice, ginger $90 \mathrm{~g}, 90 \mathrm{~g}$ of 12 pieces, $60 \mathrm{~g}$, big jujube efficacy for muscle functions, to reconcile camp guard, can also be used as a basic prescription for the treatment of other diseases and other diseases. such as the following: (1) Add Houpu 60g, almond fifty, jieji functions of dispelling wind, gasp for;(2) Increase the amount of the single twig, cassia twig and $150 \mathrm{~g}$, namely Gui Gui Tang, reuse of cassia twig, intended to Wen Tong Xinyang, in order to make kidney water, flush down the inverse;(3)The prescription of cassia twig to peony and Aconite Decoction on the sun card table mistakenly and evil injury Yang invaginations of the pathogenes is and the addition and subtraction, Yang if the injury is not heavy, can be removed if the injury is heavy Yang peony, resulting in "cold" disease, need to add temperatu re by Fuyang aconite;(4) Based on the reuse of Guizhi Decoction plus peony Xiaojianzhong decoction is composed of maltose, sweet and sour sweet combination prescription can regulate Yin and Yang.

The application of Guizhi Decoction to maltose, licorice, jujube sweet to health priorities, with Guizhi, Xin Yang Laitong regulating Wei ginger, with peony acidity and camp is pain, replenishing spleen progenitor, provides an important basis for the clinical medical treatment of offspring spleen and stomach diseases. The above party is based on square chmical process, total herbs are not many, but reflects the subtleties of the classic prescription, fully demonstrated the characteristics of a person's diagnosis and treatment of one party.

\subsection{Traditional Chinese Medicine application features - páo zhì}

The drug is according to the condition to use, improve the efficacy, reduce toxic ity must be considered is the focus of medication,so drug processing in this action can not be ignored.At the same time, taking drug treatment regimens for efficacy of increase or decrease also have an important influence.

If the drug dosage is two to six times the clothing and a variety of methods. The specific choice of drug medication, and according to the specific characteristics of pathogenesis and disease. The meal served drug concentration, rapid effect; to decline Yang, Yin Han Sheng syndrome; for old pain, is a virtual reality of the card to see the three directions; four doses were found only with serious and complicated illness; five times, six times those seen in Angelica four inverse plus Evodia Decoction and ginger pig skin soup; take a small amount of service frequency, mainly to the drug continued constantly. From the above we can see that the application of traditional Chines e medicine is also a certain precision at all.

\subsection{The similarities between Chinese medicine and Precision medicine}

Zhang zhongjing of the han dynasty, study of huangdi neijing, the concept of appropriate due to illness better applied to specific diseases, dissolve into the specific prescription. In TCM clinical syndrome differentiation process, also need to grasp the pathogenesis in order to establish the core of treatment. Zhongjingfang spread very long. Secondly, since ancient times are in use, it compare d to the updating speed of the western medicine fast, inspire us to think about Traditional Chinese Medicine formula "precision medicine". The ancient one, due to illness condition from a certain perspective, which belongs to the category of medic ine treat ment of individual.

According to the characteristics of TCM syndrome differentiation treatment, The sick people are different, and the reaction to the disease must be different. Disease have different time, place and it will be different; Disease no matter occurrence, development, recovery and so on are not immutable and frozen, such as the SARS virus, its infectious and biological characteristics will change with the 
change of the condition such as time, space, thereby increasing the treatment difficulties, we according to the characteristics of the disease drug use, so that is the precisely reflected.

See the application from the overall concept of Western medicine, and can not be completely out of the whole concept to grasp, to comprehensive clinical examination was performed only after an antidote against the disease, at this point, medicine also need to use the whole idea as a foundation, comprehensive inspection of an antidote against the dis ease in order to better treatment of $d$ is eases.

\section{6 the difference between TCM and Precise medical}

Traditional Chinese Medicine treatment is more dependent on the method of inspection, auscultation and olfaction, inquiry, and pulse-taking and palpation, to an overall understanding of the symptoms of the patients with causes, and symptomatic treatment.Such as pulse condition, The formation of pulse sequence, and the heart, blood and body flu id are closely related.Its different change reflects the heart strong or weak, relaxation, qi-blood virtual lag changes in the three aspects.Only will be the heart of the fixed, context and the flow of qi-blood, body fluid together analysis, to reveal the ess ence of the change of the pulse, What letter what Maicai reasoned, is not like water without source.Just from this aspect of pulse, different pulse condition implicating the body all aspects of function, it is only through the overall analysis to suit the remedy to the case to achieve the purpose of treating diseases.In this aspect Traditional Chinese Medicine treatment notice or as a whole, precise prefer to individualized treatment.

\subsection{The problems encountered in the application of Traditional Chinese Medicine}

Precise medical treatment concept in a person of Traditional Chinese Medicine, syndrome differentiation and treatment aspects are reflected,also play an important role, but in the face of the overall content of property theory in the treatment of Traditional Chinese Medicine and Traditional Chinese Medicine, you can't explain it, so we should dialectical view of medicine applic ation in Traditional Chinese Medicine, can not produce a plausible theory of Traditional Chinese Tedicine treatment; for example, the Traditional Chinese Medicine about four, refers to the cold temperature Liangsi different resistance, also known as four.It reflects the vicissitudes of drugs on the body of y in and Yang, cold and heat changes the tendency. It's not just to show that medic ine is to make people feel cold or warm, and modern instruments can't solve the mystery, it not because of which a specific substance to determine the temperature of drug and cold, so that the precise idea is not all powerful.

Treatment of disease of TCM should pay attention to avoid the "no overall plan for a fundamental transformation".Such as the common cold,common have a bad cold, fever, head and pain and a series of symptoms, However, due to the different pathogenic factors and physical, it is often characterized by cold, the wind hot cold, heat, and internal heat cold and other different types of evidence, only through the through overall analysis of the patient, to distinguish clearly what kind the cold, can determine the corresponding treatment methods, to give appropriate treatment, rather than just "hot" fever of local treatment.So the treatment should start from the overall concept, with the disease treatment, flexible.

\section{Discussion}

Precision treatment has many advantages in clinical application, such as accurate positioning of the lesions, reduce the patient's pain and solve some of the complicated diseases that are difficult to be solved now. at the same time there are a lot of problems need us to solve, to better provide methods for clin ical treat ment.

With the progress of modern science and technology, precision medicine in the application process of the instrument is too serious, the traditional diagnosis method was gradually forgotten, although the inspection instrument is accurate and objective, the use of modern science and technology in the diagnosis and treatment has certain advantages, but clinical experience is more important, if the 
machine depends on the instrument test results, and can not combine the actual condition, it can lead to misdiagnosis, it is not uncommon in clinical.This tells us that if we rely solely on the instrument data and the results of the examination, the temporary removal of a lesion site, the temporary relief of the patient suffering, but the follow-up related diseases caused by this can not be without thinking. So in the face of the accurate treatment of such a deficiency, we should reflect on whether you would have a long and Traditional Chinese Medicine diagnosis and treatment technology without hesitation abandon and into the precise treatment arms.

The biggest characteristic of Traditional Chinese Medicine treatment is to grasp the patient's overall treatment, find the source for treatment.It is the wisdom of the spread of thousands of crystals, but also apply to modern disease treatment.Such as the classic "the headache medicine foot" said,this is from the meridian theory. relevant organs lesions occurred.According to the meridian courses route, starting from the overall concept, to find out the source of the disease, which can treat a range of diseases.So we should start from the whole, find the source, combination with the idea of the precise treatment to achieve twice the result with half the effort to disease treatment, better services for patients.But the doctor of Traditional Chinese Medicine treatment there is a problem, such as the treatment cycle is long, the interaction mechanis m between the drug problems such as unclear, it is also need we dialectical view.

From the above discussion we can see that in the concept of treatment of TCM due to illness condition, dialectical treatment and Chinese medicine taking method in some degree belongs to the medicine category, we can see that they are common in medical research in the future, the two complementary treatment may become a trend, but can not be a requirement for Tratidional Chinese med icine accord ing toWestern med icine routine to go. each concept all have their own characteristics, and has their own areas of expertise, in their own field to shine, to form the self characteristic of Chinese medicine, in order to go out of the country, so that more people recognized, and it is not a simple precise medical treatment concept can be carried forward in traditional Chinese medicine, advanced the concept of med icine does have merit, you can learn from Traditional Chinese Medicine, its essence, to its dregs, not blindly copy,we should use dialectical attitude to treat the precise medicine. Hope that the Traditional Chinese Medicine in the modern environment, the more far away from the ancients retained the wisdom of a better use of our lives.

\section{The articles will be quoted as follows:}

project team of outstanding scientific and technological innovation in Henan province (TCJ2014-391), the natural science foundation of Henan province (132300410019), science and technology innovation team of Zhengzhou city (131PCXTD612)

\section{References}

1. J. Zheng, W. Li, The reconsideration of precision medical. Journal of medical informatics. J. 8, (2016)

2. X.L. Ru, X.H. Chen, Zhang zhongjing for quantitative identification and accurate treatment of disease and analysis. World - the modernization of traditional Chinese medicine science and technology. J. 3,10(2015)

3. F. Liu, Y.S. Yang, The characteristics of TCM syndrome differentiation, value and significance of the author. Journal of trad itional Chinese medic in.. J. 12 (2009)

4. Y.L. Wu, Accurate cancer medic ine: the way into the future.Journal of evidence-based med icine.J. 1(2015)

5. J. Wang, China's basic medical journal of traditional Chinese med icine, J. (2012)

6. X.H. Yang, Y.N. Wen, Treatment based on syndrome differentiation of traditional Chinese medicine research progress review. Journal of traditional Chinese medicine information. J. 12(2012) 
7. Y.L. Jiao, J.C. Wang, Q. Zhang, G.X. He, China facing the opportunities and challenges in the field of precision medical. ournal of public health management in China. J. (2015)

8. F. Xiao, From the evidence-based medicine to the precise medical thinking. Chinese kidney disease research on electronic magazine. J. 1 (2014)

9. Q.M. Zhan, China the strategic needs and key tasks for the development of precision medical. Chinese nerve trauma surgery electronic journal. J. 3 (2015)

10. M.Y. He, J.L. Xia, X.D. Wang, Accurate medical research progress. Journal of clinical medicine in the world. J. (2015)

11. A.W. Wu, J.F. Ji, Implementation of precision medical opportunities and challenges. Chines e journal of clinical laboratory management electronic. J. 1 (2015)

12. X.Q. Guo, W.R. Huang, Z.M. Cai, Francis choi. Cancer precision medicine science. J. 5(2015)

13. F. Jiao, S.Y. Xie, Y.J. Li, Z.L. Li, Y.F. Yan, Accurate medicine and uncertainty. Journal of med icine and philosophy. J. 1 (2015)

14. Z.F. Jiang, Breast cancer treatment decisions: fro $m$ individualized treatment to precision medical. Chinese journal of practical surgery. J. (2015)

15. H.X. Zhang, Q.Y. He, Based on the accurate medical targeted anti-tumor drugs sensitivity prediction and its application research and development. Chinese journal of new drugs. J. (2015)

16. M.C. Gong, Accurate medical challenge to medical informatics and task. Journal of medical informatics. J. 1 (2016)

17. X. Zhang, X.G. Mao, W. Zhang, Big data era of precision medical. Chinese journal of neurosurgery disease. J. 11 (2016) 